\title{
Scalable WIM: Effective Exploration in Large-scale Astrophysical Environments
}

\author{
Yinggang Li* \\ Indiana University, Bloomington, U.S.A.
}

\author{
Chi-Wing Fu ${ }^{\dagger}$ \\ Hong Kong University \\ of Science \& Technology
}

\author{
Andrew J. Hanson* \\ Indiana University, Bloomington, U.S.A
}
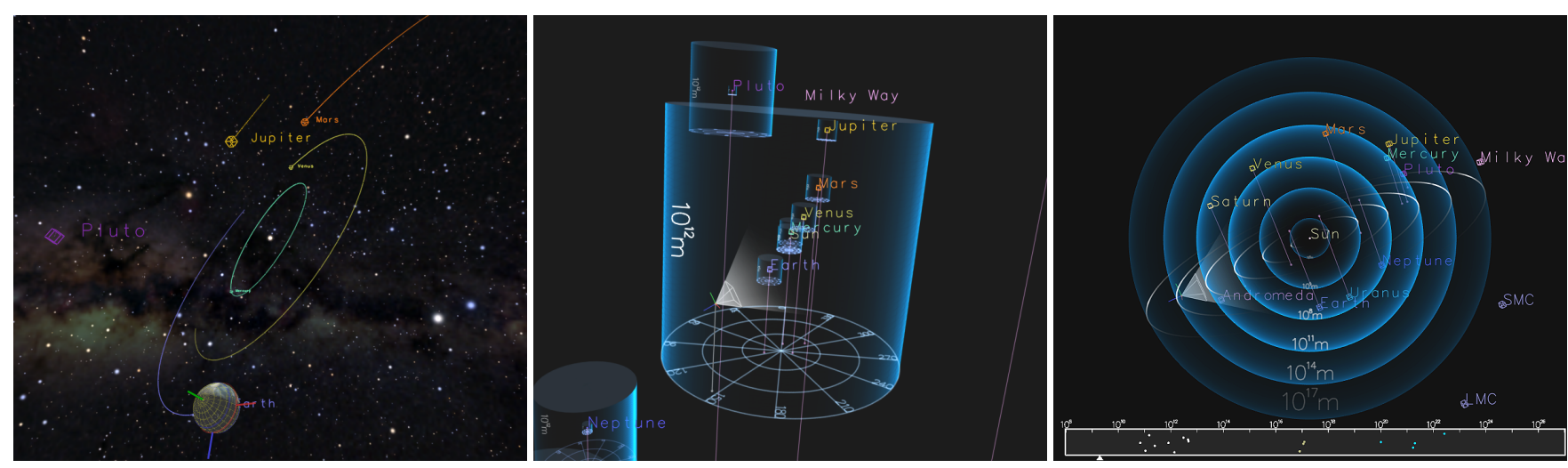

Figure 1: A scalable WIM provides not only a scale-independent 3rd person view of the current visualization, but also a 3rd person user control for us to interact with the virtual environment: Here we show a 1st person view of the solar system in a virtual Universe exploration (left), a context-cue-rich WIM offering new perspective into the local environment (middle), including a camera manipulator for view specification, and a logarithmic eye space remapping of the same scene exposing the scales in a global context (right).

\begin{abstract}
Navigating through large-scale virtual environments such as simulations of the astrophysical Universe is difficult. The huge spatial range of astronomical models and the dominance of empty space make it hard for users to travel across cosmological scales effectively, and the problem of wayfinding further impedes the user's ability to acquire reliable spatial knowledge of astronomical contexts. We introduce a new technique called the scalable worldin-miniature (WIM) map as a unifying interface to facilitate travel and wayfinding in a virtual environment spanning gigantic spatial scales: Power-law spatial scaling enables rapid and accurate transitions among widely separated regions; logarithmically mapped miniature spaces offer a global overview mode when the full context is too large; 3D landmarks represented in the WIM are enhanced by scale, positional, and directional cues to augment spatial context awareness; a series of navigation models are incorporated into the scalable WIM to improve the performance of travel tasks posed by the unique characteristics of virtual cosmic exploration. The scalable WIM user interface supports an improved physical navigation experience and assists pragmatic cognitive understanding of a visualization context that incorporates the features of largescale astronomy.
\end{abstract}

Keywords: Astrophysical visualization, large-scale exploration, interaction techniques, world-in-miniature (WIM)

Index Terms: I.3.6 [Computer Graphics]-Interaction techniques; I.6.9 [Computer Graphics]—Visualization systems and software; J.2.c [Computer Applications]: Physical Sciences and Engineering-Astronomy

\footnotetext{
*e-mail: $\{$ yinli,hanson $\} @ c s . i n d i a n a . e d u$

†e-mail: cwfu@cs.ust.hk
}

\section{INTRODUCTION}

With recent advances in modern astronomy, highly detailed spatial data far beyond our solar system have become widely accessible even to the general public. Galaxy data, stellar data, and solar system data can be easily downloaded from various astronomy websites like the JPL Planetary Data System [11] and the Simbad website [6]. Combining high-performance graphics hardware and advanced visualization techniques, we not only can achieve interactive presentations of large-scale astrophysical data in real-time, but can also explore and interact with large-scale data using various kinds of immersive VR environments. By exploiting the flexibility of visualization technologies, we can thus experience the largescale physical Universe from many different points of view.

\subsection{Motivation}

A virtual astrophysical environment intrinsically inherits two major problems from its real counterpart: scale and sparsity. The data have a huge spatial range, and yet this space is mostly empty. Besides posing challenges to the visualization implementation, these characteristics make it difficult for users to perceive the spatial scale as well as the spatial relationships among astronomical bodies in the virtual world being explored.

In our previous research, Hanson et al. [10, 8] developed largescale visualization techniques based on Power Scaled Coordinates (PSC). These methods can transparently integrate visualizations of objects at vast scales that otherwise might be limited by depth buffer and matrix transformation precision. With these techniques, we could in principle solve scalable visualization problems that encompass the heterogeneous physical Universe if it were not for the fact that the enormous amount of screen information inevitably overruns the user's ability for travel and wayfinding in the simulated virtual Universe. A new form of user interface control is thus required to enable the user to examine, navigate, and explore such a large-scale visualization space in a rapid and intuitive manner. 


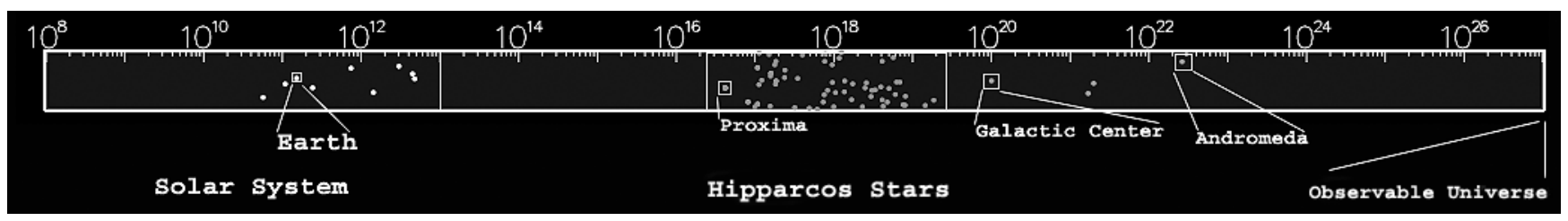

Figure 2: The logarithmic Sun-centered distance map of sky landmarks (in meters) illustrating the enormous scale range of the observable Universe: nearest star Proxima Centauri $\left(\sim 10^{17} \mathrm{~m}\right)$; Hipparcos Star Catalogue $\left(\sim 10^{19} \mathrm{~m}\right)$; Boundary of Observable Universe (Quasars, etc.) $\left(\sim 10^{27} \mathrm{~m}\right)$.

\subsection{Related Work}

The user interaction in a $3 \mathrm{D}$ virtual environment is actually the integration of two activities: travel and wayfinding.

Travel is the most common interaction technique for large-scale 3D environments, and, as such, has been the subject of considerable study. It represents motor activity controlling the position and orientation of the viewpoint. A major challenge of $3 \mathrm{D}$ travel is the mapping of control values from the input device (often only 2 degrees of freedom) to the parameters of a virtual camera model ( 7 or more, including 3 for position, 3 for orientation, and 1 for field of view). To address this challenge, a number of travel interface metaphors have been developed, including: "walking" and "flying" viewers [2], target-selection methods [20], and "world-inhand" metaphors $[19,21]$. Bowman et al. have proposed a taxonomy for classifying the components of travel techniques along with methods for evaluating their effectiveness for specific tasks [3]. The issue of speed control is particularly important for environments that are very large or sparse, and for environments with objects that exist at diverse spatial scales. Mackinlay et al. were among the first to describe a general and effective method for rapid, controlled movement in such 3D environments [15]. Celestia [13] supports an exponential zoom feature to visit nearby galaxies using extended precision arithmetic. Mine et al. $[17,16]$ investigated the problem of manipulating objects in 3D virtual environments, and proposed the automatic scaling technique to select, grab, manipulate, and release virtual objects.

Wayfinding is the tactical and strategic part of navigation that involves cognitive activity defining landmarks and paths through the environment, which can be thought of as a mental representation of environment knowledge. Darken and Sibert [5] showed that real world wayfinding and environmental design principles are effective for designing large-scale virtual worlds that support wayfinding. Applicable wayfinding techniques for virtual environments include: providing integrated 2D maps and markers; incorporating landmarks and cues into the environments; and providing a consistent and hierarchical structure for the environments.

In this work, our primary objective is to design a user interface that enables scale-insensitive interactive visualization, realized in the context of large-scale astronomical data exploration. We present a new form of World-In-Miniature (WIM) map system to overcome the travel and wayfinding problems caused by scale and sparsity. The proposed Scalable WIM design employs spatial scaling methods based on Power Scaled Coordinates (PSC) and thus permits representation and rapid transformation of positions and vectors at any arbitrary scale. Mapping a user control to the exponential power term of distance provides smooth adaptive speed control, enabling fluid travel between regions of interest that are very far apart. The Scalable WIM also provides a rich set of context cues for distance scales, motion, and orientation perception.

\section{The Study of Virtual Cosmic Exploration}

In this section, we will discuss some characteristics of astronomical data and navigation as well as justifying why a carefully designed interface is crucial for cosmological navigation in the context of astronomical wayfinding. We note that, in principle, one may differentiate two modes in which our exploratory system can be used: one is mainly pedagogical, giving the benefit of exploring the entire scale range of the Universe; the other is scientific, where in practice only, say, five orders of magnitude may enter into a single problem, but the methods could still be of value for expert exploration.

\subsection{Characteristics of Astronomical Data}

\subsubsection{Scale and Sparsity}

Astronomers mapping the Universe are challenged by a wide variety of scales. Figure 2 presents a comprehensive map of typical objects at different spatial scales in our physical Universe. The $x$ axis is the base 10 logarithm of distance relative to the Sun in meters. We can see from the map that there are 27 orders of magnitude in distance to the boundary of observable Universe: the Earth (distance scale: $\left.\sim 10^{11} \mathrm{~m}\right)$, the solar system scale $\left(\sim 10^{13} \mathrm{~m}\right)$, the nearest star to our Sun (interstellar scale: $\sim 10^{17} \mathrm{~m}$ ), the center of the Milky Way Galaxy (galactic scale: $\sim 10^{21} \mathrm{~m}$ ), and galaxy clusters (intergalactic scale: $\sim 10^{24} \mathrm{~m}$ ). Throughout these scales, space is mostly empty. In an exploration tool, we want to support a wide range of exploration capabilities, from our own solar system out to the large scale structure of galaxy groups. The challenge is thus to design mechanisms that can represent the entire observable Universe through a unified interface with proper emphasis on non-empty space.

Remark: As noted in our pedagogical film, Cosmic Clock [9], the observable Universe consists in reality of layered shells of snapshots of the Universe at progressively younger ages as the distance increases. Therefore, although we implicitly navigate through a static (or relatively static) space throughout this application, that space is not truly an accurate representation of the current Universe, but represents a set of structures seen at progressively different ages, reconstructed in a conceptual model to be our best guess of what the current Universe is like.

\subsubsection{Adaptive Reference}

The dynamic nature of celestial entities brings unique complexities to data representation and interpretation. One basic need of astronomy is to give reasonable descriptions for the positions of dynamic objects relative to each other at different scales. Different choices of reference coordinate frames exist. For instance, in the Ecliptic coordinate system, the fundamental reference plane is chosen to be the plane of the Ecliptic, i.e., the orbital plane of the Earth around the Sun, which makes sense for the evolving relationships among solar planets. Galactic coordinates, with the galactic equator as the reference plane, are used for objects beyond the solar system. Objects on the sky are clustered locally in regions that are separated by vast emptiness. To construct a cognitive map of a region and navigate using the map, users need to choose a reference system that best describes the spatial information, including locations, orientations, and the dynamics of regional features.

It is worth noting that we are restricted to capturing astronomical data from Earth, from Earth-orbiting satellites, or from spacecraft inside our solar system. Since more distant astronomical bodies are typically harder to find (due to smaller visual size measured 


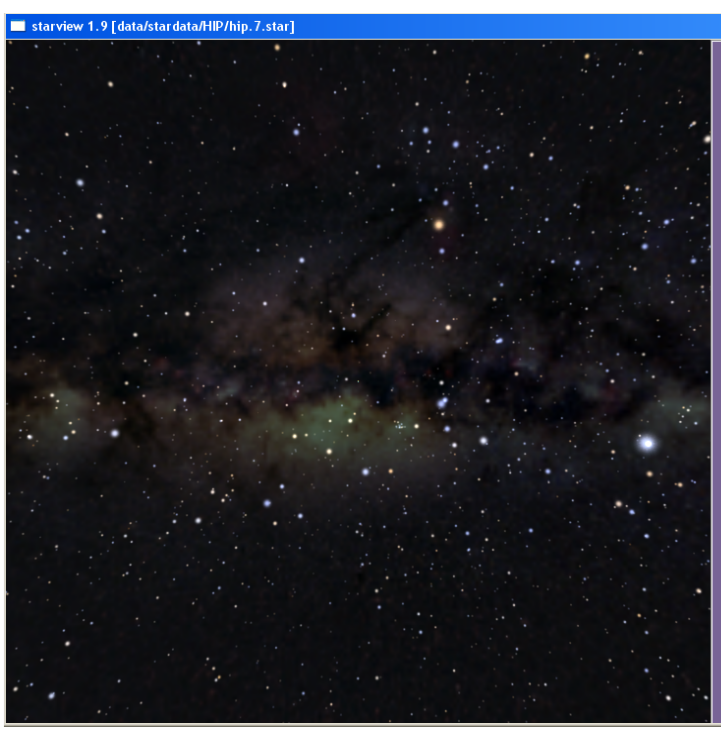

(a)

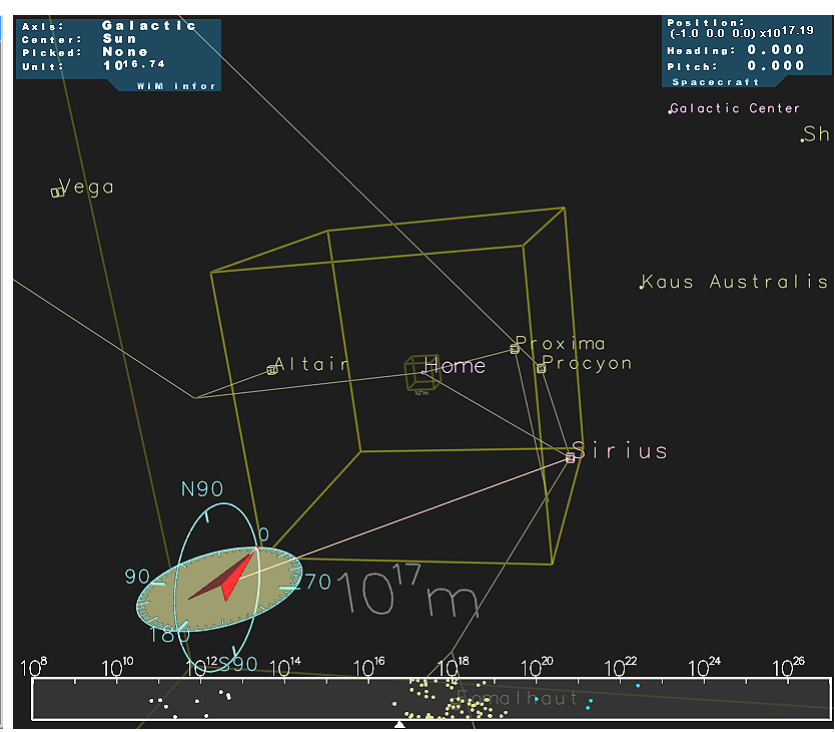

(b)

Figure 3: (a) First person view from our star exploration application. The lack of context cues limits knowledge acquisition in the first person view. (b) Scalable WIM interface enriched with landmarks and cues for scale, position, and orientation. Comparison of the two views reveals the common local context: the brightest star near the right edge is Sirius, and the dark bulge at the center is the Galactic Center (which the camera icon points to).

on Earth), most astronomical datasets have an Earth-centered bias. Thus, we effectively navigate in an Earth-centered data space. Typical datasets possessing this feature include the Hipparcos Catalogue [1] for stars and the Tully Galaxy catalog [23]. However, when we travel into unbounded deep space, the invisible Earth may not be the most intuitive choice of origin. The Sun, the Milky Way Galaxy, or simply the location of "the spacecraft" may serve better.

\subsection{Characteristics of Astronomical Navigation}

To enhance the performance of navigation tasks, we need to understand how those tasks are constructed and carried out, i.e., by constructing the navigation model for our application. The general model proposed by Jul and Furnas [12] still applies in the context of Universe exploration, but the perception, assessment and strategy selection, etc., will be very different from any daily-life virtualworld navigation model. When we seek a serendipitous exploration experience with minimal loss of context, it is imperative to take into account the complexities introduced by astronomical navigation characteristics.

\subsubsection{Adaptive Travel Speed}

We first address the adaptive speed used to travel in a virtual astronomical environment. Fans of Star Trek are familiar with the concept of "warp speed" in interstellar exploration. During the course of navigation, we need to adapt the speed for different situations. User interface designers normally associate an input device's sensitivity with a travel speed. For instance, to travel among planets inside our solar system, we may choose a certain speed for each unit of input device displacement. But if we travel with that same sensitivity through interstellar space or intergalactic space, our motion with respect to the objects of interest would be static because the distances to be covered are just too vast relative to the speed. On the other hand, if we travel at interstellar or intergalactic speed inside the solar system, we would quickly get lost in deep space and never succeed in approaching a target planet, since Galactic scale controls do not provide sufficiently fine control at solar system scales.

The practical implementation of adaptive speed of course exceeds the speed of light, which limits actual physical travel (e.g., sunlight takes about 8.5 minutes to reach us on Earth, while light from our nearest star system, the Alpha Centauri triple star system, takes about 4.3 years to reach us). If we used physically realistic travel constraints, the travel time to objects of interest would simply be too long. Therefore, we follow the common practice in cosmic exploration and allow arbitrary speeds during navigation, motivated by sheer practical need.

\subsubsection{Context Awareness in Sparse Space}

According to the theories of cosmology, the Universe has gone through cosmological eras dominated by radiation and matter, and has now become a vacuum-dominated Universe. In this vacuumdominated era, astronomical bodies are tremendously far apart, and this exacerbates the losing-spatial-context problem during wayfinding.

In daily-life environments, we can usually identify landmarks with salient cues such as appearance, structure, or labels. For proficient navigation, we organize these landmarks together in a mental representation of the environment context. However, the space separating astronomical objects is essentially empty and unbounded. The user's perception could be tricked by empty background space or the fact that a very distant object can be more visible than a nearby one. For example, the apparently aligned stars in the Big Dipper are at very different distances. It is very hard for navigators to perceive the spatial context around the navigation regions until they finally reach the destination scale range. (See Figure 2 for scale range examples). In addition, the high similarity of appearance among astronomical objects requires the introduction of artificial landmarks such as constellations. Figure 3 illustrates the need for navigation assistance in interstellar space.

\section{INTERFACE DESIGN AND IMPLEMENTATION}

While a selection of the challenges mentioned in the previous section have been addressed in other astronomy simulation software (e.g., Celestia, Stellarium, Open Universe, etc.), little effort has been devoted to integrating these two conflicting goals: (1) maintaining fast interactive performance through vast spatial scales without being overwhelmed by information encountered between 

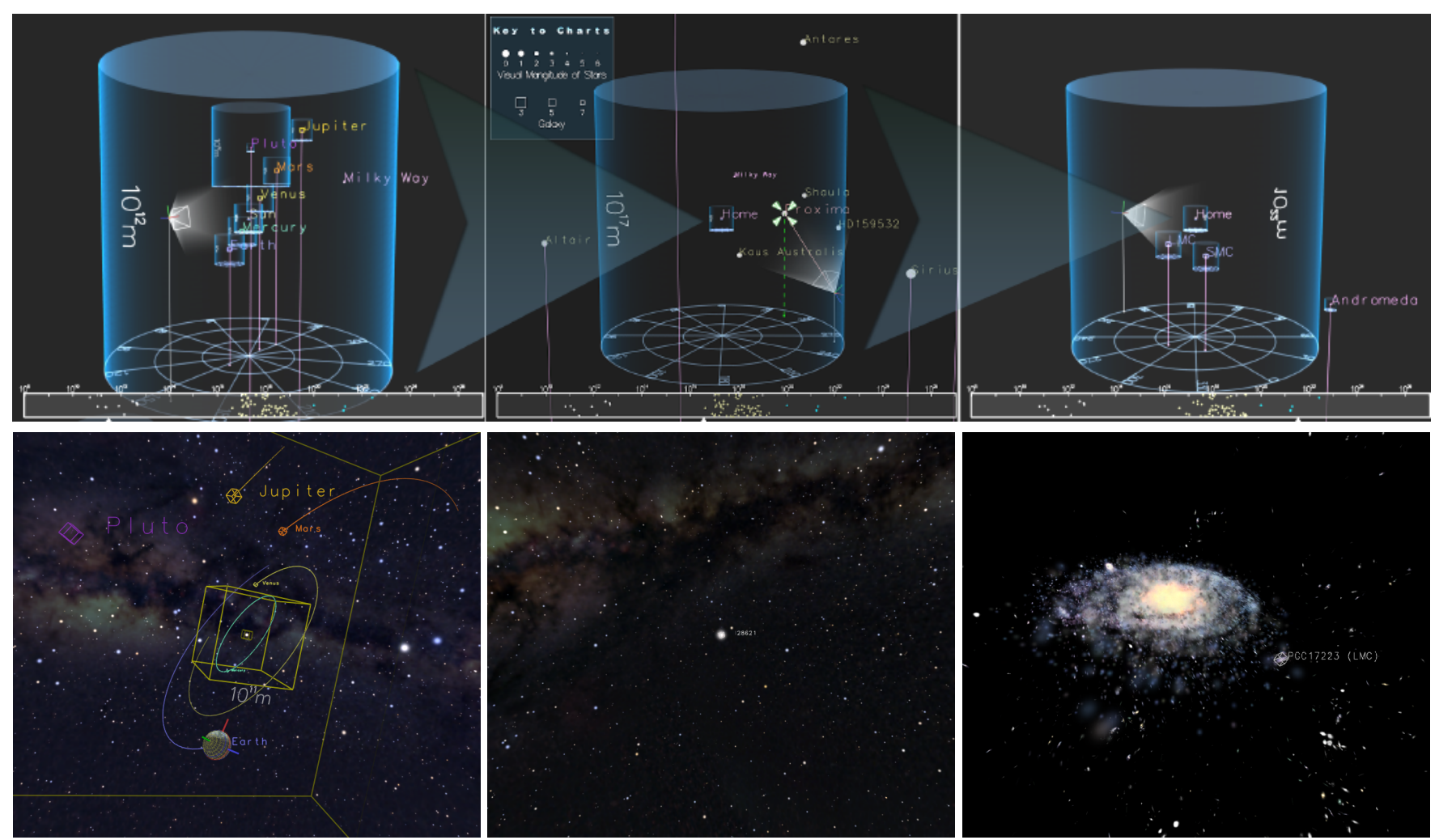

Figure 4: (Top row): Power spatial scaling in a Scalable WIM map: A journey from the inner planets at $10^{12} \mathrm{~m}$ (left), to the nearest star, Proxima Centauri at $10^{17} \mathrm{~m}$ (middle), and finally to the Local Group of our Milky Way galaxy $10^{22} \mathrm{~m}$ (right). (Bottom row): Scenes along the journey as seen from the first person view.

destinations; (2) maintaining enough context awareness using distinct spatial landmarks to enable further meaningful interaction.

We designed the Scalable World-In-Miniature (WIM) environment as a user interface component supported by the PSC-based visualization framework for modeling and rendering with large spatial scales [8]. (Maps are intensively used in games to enhance navigation performance.) The World-In-Miniature (WIM) [19] method, as a $3 \mathrm{D}$ virtual map, is a scaled-down iconic version of the virtual world. The WIM approach provides a third-person map perspective to supplement the immersive presence in a virtual world, so that users can quickly acquire the context of the virtual environment in abstract form, and can directly control their movements through the WIM's display of context information for the given camera viewpoint. The key techniques given below are incorporated into our design to extend the WIM's capability to aid large-scale cosmic exploration.

\subsection{Power Spatial Scaling in WIM}

When the extent of a large-scale dataset exceeds the hardware representable bounds, one conventional WIM design is to ignore the physical scale, and to artificially adjust the spatial scale in the eye space by making the objects arbitrarily closer or bigger so that everything becomes renderable at a chosen series of ad hoc physical scales. Another approach is to divide a large virtual world into subregions and switch "gears" for separate scaling treatments [5]. Both approaches are sufficient for exploring pre-rendered material. In many existing virtual cosmic exploration applications, one can detect noticeable discrete motion errors at large scales, indicating that the methods for handling spatial scales do not consistently avoid scaling errors. With the growing demand for real-time interactive control and exact scientific accuracy in visual exploration systems, the need for a comprehensive approach that handles all scales in a single context becomes apparent.
As the name suggests, the proposed Scalable WIM is a largescale 3D miniature map equipped with spatial scaling control. Our overall large-scale rendering strategy is implemented via the PSC representation and transformations presented in our related work $[8,10]$. In short, 3D coordinates and vectors are represented by a unit directional term and an exponential distance term (usually base 10 ), so that we can express coordinates ranging from super-galactic to sub-atomic scales in a uniform fashion. Consequently, the translation component in the WIM transformation can be generalized as follows in base 10:

$$
T_{W I M}=\left(T_{x}, T_{y}, T_{z}\right)^{t} \times 10^{s_{W I M}},
$$

where $s_{W I M}$ is the relative spatial scale associated with the current eye space. One abstract unit in the WIM corresponds to $10^{s_{W I M}}$ physical units (typically meters) in the physical Universe. Adjusting $s_{W I M}$ exponentially scales the WIM space accordingly. The camera setting is unaffected during the change. In other words, we scale the entire simulated Universe's data relative to the eye space.

With power-law spatial scaling, the WIM world can be easily zoomed to different levels and allows users to efficiently explore surrounding spatial context at different scales. If we map the mouse/keyboard input space to the exponential scale $s_{W I M}$, the 3rd person viewpoint controlled by the user can move freely to a certain "current" power scale while the physical data set is scaled linearly relative to this scale. For example, when the camera is pulled from a location at scale $10^{13} \mathrm{~m}$ to a location at scale $10^{14} \mathrm{~m}$, the data at source scale will be continuously shrunk down to one tenth of the original dimension in the eye space units, and data at the destination scale will be scaled into one unit in the eye space.

The effect of continuous power spatial scaling is a smooth and fast context transition across large scale ranges. Entities at much lower scales would occupy less than a pixel at the fixed scaling 
center, and entities at much higher scales would be outside of the WIM viewing frustum. Therefore, the user can focus on the landmarks within adjacent local scales. Power spatial scaling is also accurate: precise positioning and direct manipulation of objects can be carried out in the Scalable WIM. Figure 4 shows Scalable WIM snapshots at different levels along a navigation path from the Solar System to the Local Group. Note that the dominant power cue in the left image is within the invisible center of the cue in the right image.

\subsection{Landmarks and Context Cues}

\subsubsection{Landmarks}

The primary manipulator representing the navigator takes two forms in the WIM: a camera icon for view specification (see Figure $3 \mathrm{~b}$ ) or a 3D compass for orientation reference (see Figure 4). The user can quickly change the 3rd person viewpoint in the WIM by rotating and scaling the entire miniature world without destroying the view established in the 1st person immersive world. Such a WIM "fly-by" gives the user a better understanding of the space that the camera inhabits and assists accurate view specification. To acquire orientation-specific knowledge, we project the celestial sphere onto a sextant-like compass located at the camera position. Each direction away from the viewpoint will intersect the compass sphere in one unique point, and positions of stars and other celestial objects can be measured in angular coordinates on this virtual sphere. The two great circles denoting the equator and the prime meridian in the chosen coordinate system are marked for reference.

Celestial landmarks are often indistinct or hard to remember. To enhance wayfinding without overwhelming the user with artificial screen annotations, we employ selected meaningful symbols to focus user attention on landmarks or to guide the user through the visualization in a particular order. Text labels and icons are attached to 3D landmarks represented in the WIM to resolve similar appearances. Text collision and occlusion strategies are applied to deal with visibility issues when clutter occurs. A floating Key-To-Charts can be overlaid on top of landmarks for convenient reference.

Object marking and picking are synchronized in both the WIM and the immersive view. The user can associate the real visual simulation to a more meaningful textual reference highlighted simultaneously in the two views. The camera manipulator can be quickly rotated to align with the line of sight to the picked landmark in the WIM, and a real-time update of the view takes place immediately in the 1st person immersive view.

\subsubsection{Power cues}

To enhance awareness of the spatial scaling effects, we use one of three different $3 \mathrm{D}$ power cues to show the spatial scale:

- Power Cube: Corner edges are the same as the Cartesian frame of the chosen coordinate system. This delivers good depth context in stereo, see Figure $3 b$.

- Power Cylinder: Lid is parallel to the equatorial plane of the chosen coordinate system. This can show proximity projection within the extent of the cylinders, see Figures 4 and 5.

- Power Sphere: Illustrates radius and direction from the world center, and matches the cognitive Universe model of nonexpert users, see Figures 6 and 7.

Each power cue assumes a power-of-10 size in the exponential scale, similar in spirit to the 2D spatial squares shown in the "Powers of Ten" movie [4].

Power cues are both centered and nested. First, the user has the option to decide where to center a power cue: the origin of the reference coordinate system, the camera (the traveler), or at a selected landmark. If centered on the coordinate origin, power cues are structured as a geocentric map; if centered on spacecraft, they become an egocentric map. During spatial scaling, the spatial context related to the center is readily perceived when power cues shrink towards or expand out from the center. In this sense, power cues provide regional anchors with which a bounded naive search can be conducted in the vicinity of the subject of interest. Secondly, we can nest multiple sub-cues at lower scale levels within a common power cue at a higher level. This cue-in-cue display organizes objects into a coherent structure and enhances the ease with which objects can be recognized. This artificial structure introduces legibility to the large, sparse virtual space, similar to the role of walls and floors in small bounded environments.

During the course of navigation, we adaptively change the reference coordinate system to the one best describing the local spatial context, as mentioned in the astronomical data section. Power cues are rotated as needed to align with the chosen coordinate system orientation.

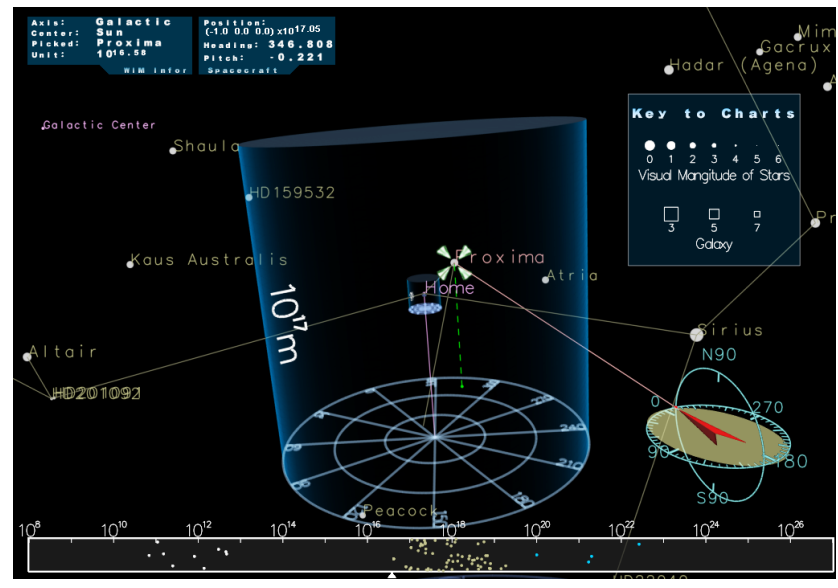

Figure 5: Context cues and landmark representations on the WIM interface: the power cylinder cue at $10^{17} \mathrm{~m}$, landmark icons and labels, MST edges, sextant-like compass and overview map.

\subsubsection{Proximity Cues}

When the user navigates among stars at an interstellar scale or among galaxies at an intergalactic scale, there are several thousand targets in proximity; though we can project these onto a plane using the power cylinder cue, or dynamically vary their rendering sizes according to their proximities from us, it is often difficult to visualize the spatial context and relationships.

We therefore provide the minimum spanning tree (MST) method in the Scalable WIM to effectively represent the changing spatial domain in proximity to the camera. When star data are loaded at system launch time, we first construct a fully connected graph in which the edge cost is defined as the distance between the associated star pair. Prim's algorithm is applied to determine the MST structure. During interaction, part of these pre-built MST edges dynamically fade in/out according to their proximities to the moving camera viewpoint. The user can better perceive the spatial relationships among cluttered entities when the neighbors are visually connected and highlighted. Figure 5 shows a star rendering example incorporating edges from the MST: Sirius and Procyon (right margin) are in close proximity to the traveler, while Hadar of the Centaurus constellation is far away despite its high visibility.

\subsubsection{Overview Map}

We use spatial scaling to show continuous sequences of 3D maps at different scale levels. At each level, however, the detailed context may be illegible for the lower or higher scale regions. This 
may not be desirable in a wayfinding task in which the target is invisible within nested power cues. We provide a $2 \mathrm{D}$ overview map of the Universe (see the map at the bottom of Figure 5), similar to Figure 2, as a global context cue. Due to the overwhelming number of objects, we only take a slice of the sky near the Equatorial plane ( \pm 5 Declination), and flatten the slice into a $2 \mathrm{D}$ chart. The coordinates of objects on this chart are: $(r, \theta)$, where $r=\log \left(\sqrt{x^{2}+y^{2}+z^{2}}\right), \theta=\tan ^{-1}(y / x)$. In short, we collapse the Declination and use the logarithm of the distance to the origin and RA (right ascension) as 2D map coordinates. This map shows the current WIM scale as a slider over the entire representable scale range. It provides a handler for fast and accurate WIM power spatial scaling, and displays the local distribution of virtual entities.

\subsection{Logarithmic Space Mapping}

The exploration of a very large dataset requires the ability to see all the data at once in a variety of different views and scales; a properly maintained global context provides a hierarchical means to facilitate knowledge acquisition and analysis. If the full context cannot fit within the graphics eye space, how do users see from a map the detail they need to navigate while maintaining a sense of scale and location with respect to the entire space?

To deal with this issue, we apply the logarithmic mapping technique used in the 2D overview map and in Figure 2 to the WIM eye space mapping. Recall that in the power spatial scaling mode, the entire world space is linearly scaled relative to the "current" WIM scale. We now introduce an overview mode where the real world space is mapped logarithmically relative to a certain WIM scale. In this way, we can fit the entire observable Universe up to the scale of $10^{27}$ meters in 27 units of eye space. Note that the logarithmic space mapping is distorted, but it does provide a new look at the world in its entirety, rather than just parts or regions. Figure 6 is thus able to display the solar system planets, nearby stars, and galaxies all one in WIM.

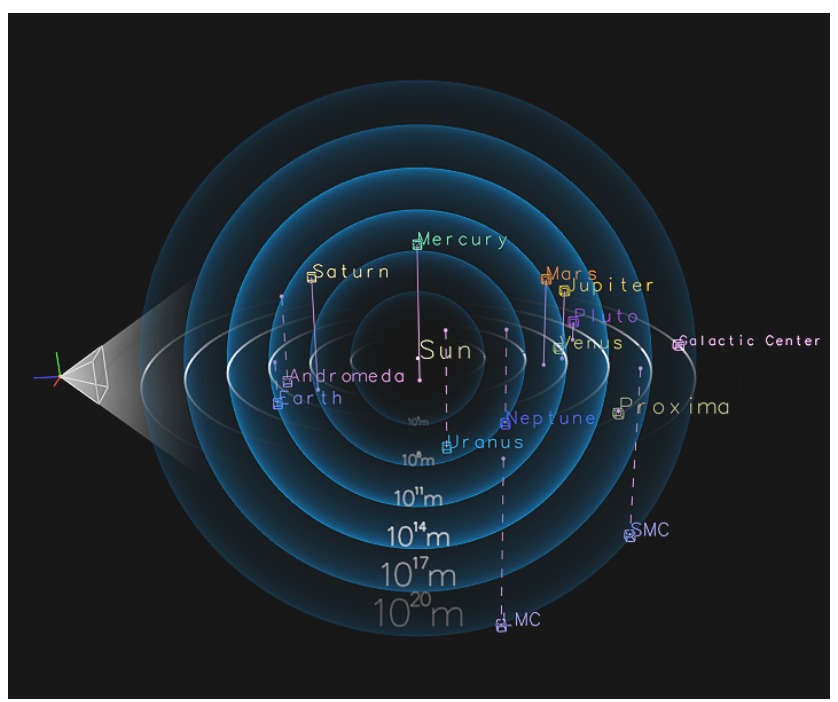

Figure 6: Log Sky: Showing landmarks of the sky at a single glance with logarithmically mapped space.

\subsection{System Implementation}

The Scalable WIM is developed as a portable map system in the navigation module for a variety of large-scale interactive applications. In particular, it is built on top of the power scaled coordinates module for scale-insensitive representation and transformation, but is independent of the modeling and rendering modules in the overall system architecture. Furthermore, we have an interface module to connect the rendering and navigation modules within the application layer; hence, the Scalable WIM can establish a viewing frustum (3rd person view) different from the one in the rendering module (1st person view), and the two modules can notify each other and synchronize the changes to camera parameter settings and landmark locations.

In practice, we implemented the Scalable WIM using OpenGL for the rendering, FreeGLUT for the window management, and GLUI for the UI widgets. The simulated astronomical images we present in this work are based on data that include the solar system data from JPL NASA [11], star data from the Hipparcos catalog [1] and galaxy data from Tully [23]. The rendered images and the accompanying video were produced on a Pentium IV PC with a 2.8 $\mathrm{GHz}$ processor and an NVidia GeForce FX5200 graphics board. With the highest-quality photorealistic rendering turned on and a hundred thousand stars loaded in the database, the immersive view can be updated at a speed of at least $10 \mathrm{fps}$, while the user interaction with a modeless WIM map runs at about $25 \mathrm{fps}$, thanks to the lightweight, symbolic, and selective landmarks in the WIM.

\section{USER INTERACTION THROUGH SCALABLE WIM}

Context viewing, landmark selection and camera manipulation are the three basic WIM operations that the user may engage in. When the user explores the immersive environment, the most common task is to "fly" through regions of interest. The user reaches into the WIM to carry out a navigation task through a sequential combination of operations. With extra support from spatial scaling and context cues, our Scalable WIM enables three aided navigation models for specific travel and wayfinding tasks.

\subsection{The Select-and-Go Model}

In a primed search task, the user knows the location of the target and can move there directly through a select-and-go navigation mode. Users can select a target object through a menu or by mouse-picking in the WIM, and the system smoothly interpolates the user's viewpoint to the target. This is a conventional user model available in other applications such as Celestia and the Digital Universe.

Because the user is transported directly to the target, there is still a nontrivial loss-of-spatial-context problem. In our select-and-go model, this problem is alleviated by the usage of Tethering-targetcentered power cues. Nested power cues are displayed and centered at the source object to reveal its surrounding context when the user is departing from it. Just before one reaches the power cues that enclose the destination object, the source power cues fade out and the set of power cues at the new target fade in. This method improves context transitions through empty space.

\subsection{The Spatial Scaling Model}

As mentioned in the implementation section, the power scaling method helps present data at their most appropriate scales around the 3rd person viewpoint in the Scalable WIM. When dealing with large-scale navigation, power spatial scaling enables the user to make fast transitions across different scales. By mapping the exponential parameter $s_{W I M}$ to the user input (mouse or keyboard), the user can readily reach the destination (the $s_{W I M}$ term is adjusted interactively to the desired scale corresponding to the landmark we want to reach). The user continuously senses a reasonable abstract (rescaled) travel speed since all models are scaled relative to a fixed camera scale. In this way, adaptive speed control can be achieved without adjusting input control sensitivity.

Most users have limited a priori knowledge of a destination's exact location, but in practice they may know a well-defined astronomical region for a target. The location of Andromeda, for instance, may be known as "in the Local group at $\sim 10^{24} \mathrm{~m}$." The user may use the spatial scaling model as an expedient way to transition 


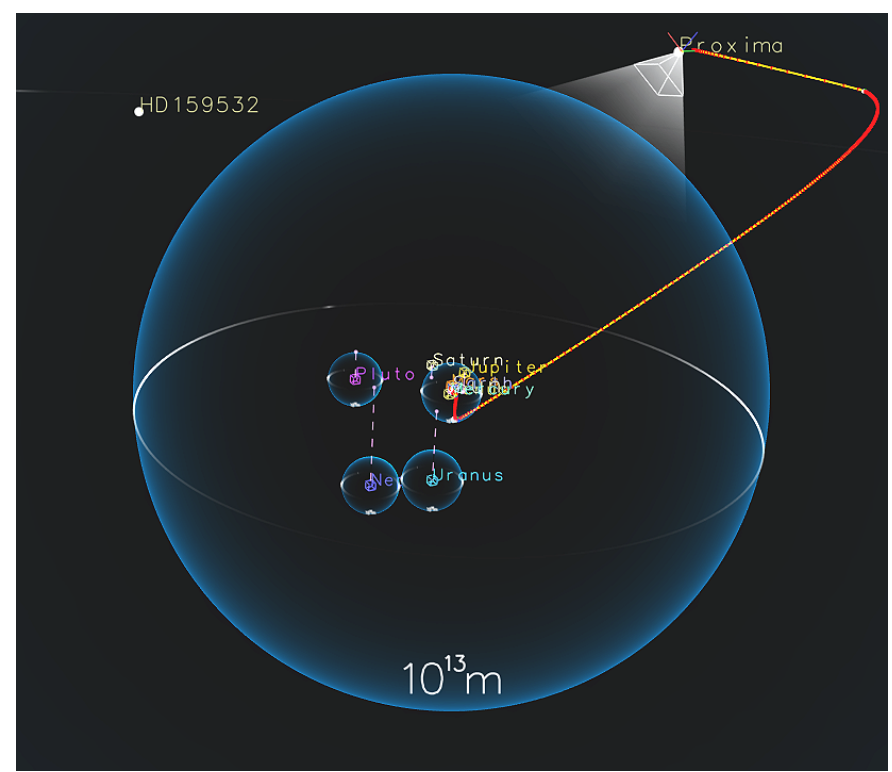

(a)

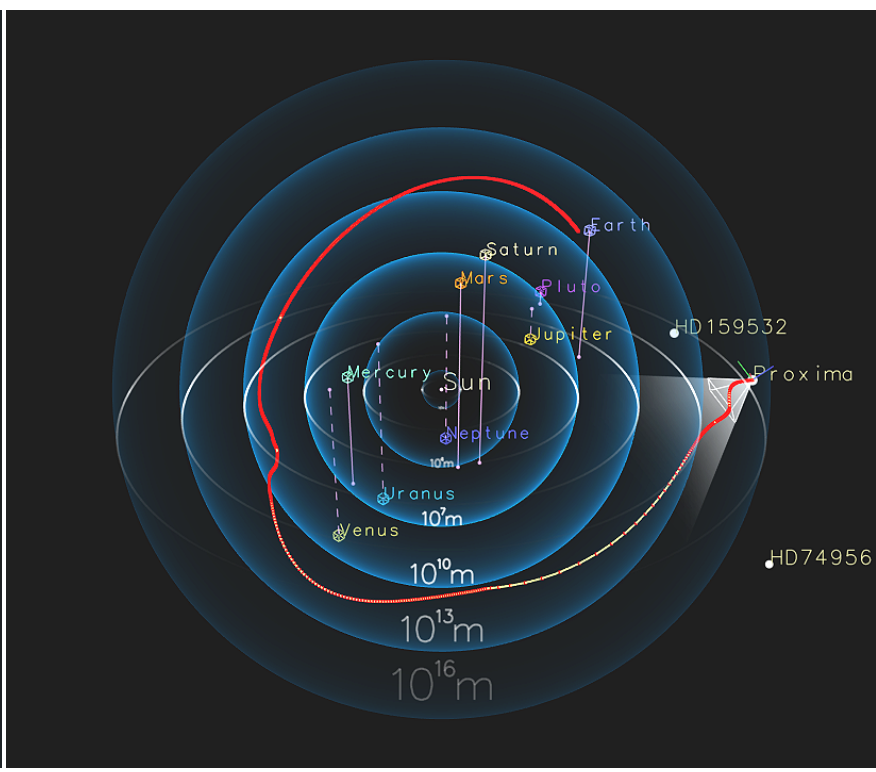

(b)

Figure 7: Planning a travel path from Centauri Proxima $\left(10^{17}\right)$ to Earth $\left(10^{11}\right)$ : (a) Path planning using continuous spatial scaling. Path detail is invisible at higher or lower scales. (b) The same path seen in logarithmically mapped eye space as a complete entity.

to the target scale, and then can conduct exhaustive unaided search within that target region to find the precise target location.

\subsection{The Path-based Model}

By specifying a series of landmarks, the user can create a flight along an interpolated path passing all the landmarks in sequence. The main advantage of using navigation paths for astronomical exploration lies in the fact that the user can choose to stay on the path, and let the system guide the way, or can stop at any point of interest on the path, detach from the path and explore local context, then continue back on the guided path.

The path offered by the path-based model must span enormous scales to support guided tours through the cosmos. Since context details are invisible at dissimilar scales in the linear space mapping, the user has to constantly conduct power scaling in order to plan and check a path between successive landmarks. The overview mode enabled by logarithmic space mapping is the natural choice to plan the path as a whole. As shown in Figure 7, the path planned in linear mode will take a different shape compared to the logarithmically mapped overview mode.

\section{Conclusion ANd Future WORK}

Scale and context are major issues in the communication of astrophysical information to the public and in astronomy education in general. While scant attention has been paid to astronomical scales, microscopic scales, with very similar issues, are the subject of wide interest in education (see, e.g., the Nanoscale Science Education web page [24] or Tretter and Jones [22]). The completion and analysis of a systematic user study is planned for the near future to quantify the nature of users' experiences at various levels of expertise with the Scalable WIM vs non-WIM methods. Our own qualitative experiences within the laboratory setting have the following features:

- Without the Scalable WIM, even experienced astronomy visualizers can easily become lost when trying to navigate a transition from, e.g., the Solar System to the location of Alpha Centauri, one of the nearest stars. Employing the WIM almost completely eliminates the possibility of becoming confused about the spatial context.

- Exploration of an environment is typically tedious in unassisted exploration methods. Without a feeling of where one has been and a mental image of what one has yet to see, motivation to seek out an exhaustive list of interesting objects is difficult to sustain. The Scalable WIM motivates long-term explorations, apparently because the mental catalog of what to go look at next is easier to keep in mind; the time voluntarily taken in a single session to explore a virtual world is typically longer and motivation is sustained at a higher level with the Scalable WIM interface.

In summary, we have presented a user interface design for exploring the physical Universe despite its sparsity and wide range of scales. The 3D Scalable World-In-Miniature map incorporates continuous spatial scaling and scale mapping to assist navigation through large-scale virtual spaces. With the aid of cues for wayfinding, position and orientation are properly maintained, and the logarithmic spatial context mapping further enhances the cognitive experience and global context awareness.

Our major focus to date has been on incorporating the design concept of the Scalable WIM in an effective user interface for virtual cosmic exploration. A great deal of research still needs to be done on applications supporting wide ranges of spatial scale environments such as quantum scales and nanoscales, as well as astrophysical scales. In fact, the improvement of concepts of scale is one of the four unifying science education themes recommended by the American Association for the Advancement of Science [7]. We hope to further develop design methodologies focused on elements and organizational principles of teaching scale hierarchies, similar to the Urban environment analysis done by Lynch [14] and the architectural analysis by Passini [18]. We also hope to examine what existing cognitive frameworks are held by non-scientists and scientists with respect to scaling concepts. Finally, we would like to explore the possibilities of integrating a detached scalable WIM into a tablet or portable devices for flexible control in immersive virtual reality environments. 


\section{ACKNOWLEDGEMENTS}

We would like to thank Mario Juric for his input on the issues involved in mapping the Universe, Catherine Pilachowski and Priscilla Frisch for their helpful astronomy input, and the referees for their insightful comments and suggestions. We are grateful to Silicon Graphics Corporation for supporting a portion of the work leading to this paper. This research is partly supported by the Research Grants Council of the Hong Kong Special Administrative Region, under RGC Earmarked Grants (Project No. HKUST612505).

\section{REFERENCES}

[1] E. S. Agency. The Hipparcos and Tycho Catalogues, ESA SP-1200, 1997. Available at http: / / www.rssd.esa.int/Hipparcos/ catalog.html.

[2] G. Bell, R. Carey, and C. Marrin. The Virtual Reality Modeling Language (VRML), version 2.0, 1996.

[3] D. Bowman, D. Koller, and L. F. Hodges. A methodology for the evaluation of travel techniques for immersive virtual environments. In VR. Journal of the Virtual Reality Society, 3:120-131, 1998.

[4] C. and R. Eames. Powers of Ten, 1977. 9 1/2 minute film, made for IBM.

[5] R. P. Darken and J. L. Sibert. Wayfinding strategies and behaviors in large virtual worlds. In Proceedings of the SIGCHI conference on Human factors in computing systems, pages 142-149, 1996.

[6] C. de Données astronomiques de Strasbourg. Simbad astronomical database. Website: http://simbad.u-strasbg.fr.

[7] A. A. for the Advancement of Science. Benchmarks for science literacy. New York: Oxford University Press Inc., 1993.

[8] C.-W. Fu and A. J. Hanson. A transparently scalable visualization architecture for exploring the Universe. IEEE Transactions on Visualization and Computer Graphics, 2006. to appear.

[9] A. J. Hanson and C.-W. Fu. Cosmic clock, 2000. Siggraph Video Review, vol. 134, scene 5.

[10] A. J. Hanson, C.-W. Fu, and E. A. Wernert. Very large scale visualization methods for astrophysical data. In W. de Leeuw and R. van Liere, editors, Data Visualization 2000, pages 115-124. Springer Verlag, 2000. Proceedings of the Joint EUROGRAPHICS and IEEE TCVG Symposium on Visualization, May 29-31, 2000, Amsterdam, the Netherlands.

[11] N. Jet Propulsion Laboratory. Planetary data system, 2003. Website: http://pds.jpl.nasa.gov.

[12] S. Jul. and G. W. Furnas. Navigation in electronic worlds: A chi 97 workshop. In SIGCHI Bulletin, pages 44-49, 1997.

[13] C. Laurel, C. Weisbrod, F. Schrempp, and C. Teyssier. Celestia, 20012006. http: / / www. shatters. net/celestia.

[14] K. Lynch. The Image of the City. MIT Press, 1960.

[15] J. D. Mackinlay, S. Card, and G. Robertson. Rapid controlled movement through a virtual 3D workspace. In SIGGRAPH '90 Proceedings, pages 171-176, 1990.

[16] M. R. Mine. Exploiting proprioception in virtual-environment interaction. Technical report, University of North Carolina, 1997. UNC Computer Science Technical Report TR97-014.

[17] M. R. Mine, J. F. P. Brooks, and C. H. Sequin. Moving objects in space: Exploiting proprioception in virtual environment interaction. In SIGGRAPH '97 Proceedings, pages 19-26, 1997.

[18] R. Passini. Wayfinding in Architecture. New York: Van Nostrand Reinhold Company Inc., 1984.

[19] R. Pausch, T. Burnette, D. Brockway, and M. E. Weiblen. Navigation and locomotion in virtual worlds via flight into hand-held miniatures. In SIGGRAPH '95 Proceedings, pages 399-400, 1995.

[20] J. S. Pierce and R. Pausch. Navigation with place representations and visible landmarks. In IEEE Virtual Reality '04, pages 173-180, 2004.

[21] R. Stoakley, M. J. Conway, and R. Pausch. Virtual reality on a WIM: interactive worlds in miniature. In Proceedings of the SIGCHI conference on Human factors in computing systems, pages 265-272, 1995.

[22] T. Tretter and M. G. Jones. A sense of scale: The importance of size. Science Teacher, 70(1):22-25, 2003.
[23] R. B. Tully. Nearby Galaxies Catalog. Cambridge University Press, 1988.

[24] The nanoscale science education research group. http://ced. ncsu.edu/nanoscale/scale.htm. 